\title{
One-step electrosynthesis of multi-walled carbon nanotube/poly-ortho-aminophenol composite film and investigation of its electrocatalytic properties
}

Narges Ajami ${ }^{*}$ and Niloufar Bahrami Panah

\begin{abstract}
In this study, one-step electrochemical polymerization method was developed for the fabrication of multi-walled carbon nanotubes/poly-ortho-aminophenol (MWCNTs/PoAP) composite film to detect oxytetracycline. The fabricated nanocomposite shows good stability and enhanced electrocatalytic ability for the detection of oxytetracycline. The resulting composite can be used as electrode material in an electrochemical capacitor. Scanning electron microscopy and electrochemical methods have been used to characterize the MWCNTs/PoAP nanocomposite film.
\end{abstract}

Keywords: Linear sweep voltammetry; Multi-walled carbon nanotube; Oxytetracycline; Poly ortho aminophenol

\section{Background}

Conducting polymers are important materials of considerable interest in many fields including corrosion inhibitors, batteries, organic electronics, electrochromic devices and sensors [1-6]. Among Conducting polymers, poly ortho aminophenol, has been research focus $[7,8]$, which is due to its good redox reversibility, unique doping characteristics, good stability, and its high conductivity. Multi-walled carbon nanotubes (MWCNTs) have chemical stability, highly accessible surface area, unique structure, narrow distribution size, and low resistivity [9-13]. It has been shown experimentally that the introduction of carbon nanotubes (CNTs) into a polymer matrix improves the electric conductivity as well as the mechanical property of the original polymer matrix [14-16]. Oxytetracycline is one of the most common tetracyclines that are widely used for the treatment of infectious diseases [17]. Modified electrodes are being used continuously in the voltammetric determination of organic compounds because of their efficiency and the selectivity that can be obtained by varying the modifier. Herein, we describe a simple strategy for the aligning of disordered CNTs within

\footnotetext{
* Correspondence: n_ajami@pnu.ac.ir

Department of Chemistry, Payame Noor University, PO BOX, 19395-3697, Tehran, IRAN
}

\section{倠

the conducting polymer matrix by in situ electropolymerization using an ionic surfactant as the supporting electrolyte. In this study, the modified electrode was fabricated successively by electropolymerization of orthoaminophenol (oAP) in the presence of dodecyl benzene sulfonic acid sodium (DBSA) onto a platinum electrode (PtE) surface. The prepared electrode was characterized by linear sweep voltammetry, cyclic voltammetry, and scanning electron microscopy (SEM) techniques. It exhibited excellent electrochemical response to oxytetracycline with good stability and sensitivity and featured with a low detection limit and wide linear range.

\section{Results and discussion}

Characterization of the modified electrode

Figure 1 shows the cyclic voltammograms of the electropolymerization of ortho-aminophenol monomers in the presence of MWCNs. The effect of DBSA, as an anionic dopant, on the electropolymerization of the polymer in aqueous solutions has been investigated. DBSA increases the conductivity of the polymer [7]. The broad peak on the first positive sweep (Inset of Figure 1) between 0.61 and $0.75 \mathrm{~V}$ is due to the oxidation of the hydroxyl group of oAP and amino group, which leads to the polymerization of the monomer [18]. In the second cycle, 
a pair of redox peaks appears at 0.34 and $0.31 \mathrm{~V}$, which related to the formation of a cyclic dimmer of the polymer, the 3-aminophenoxazone (3APZ). As it can be seen in Figure 1, during continuous cycling, the current response of this pair of redox peaks diminishes slowly, and at the same time, another redox peak appears between 0.1 and $0.23 \mathrm{~V}$. This pair of redox peaks is due to the oxidation and reduction of PoAP, indicating the formation of PoAP [19]. Therefore, $3 \mathrm{APZ}$ plays the role of a monomer in the formation of PoAP as evident from the disappearance of the system at $0.34 \mathrm{~V}$ and corresponding growth of the polymer film between 0.1 and $0.23 \mathrm{~V}$.

The cyclic voltammograms of the PoAP film and MWCNTs/PoAP composite have a couple of strong and broad oxidation and reduction waves in a solution of 0.5 $\mathrm{M} \mathrm{NaClO} / \mathrm{HClO}_{4}$ (Figure 2). Figure 2a shows a pair of redox peaks at around $0.12 \mathrm{~V}$ attributed to the redox process of the phenoxazine units. The evanescence of a pair of redox peaks at around $0.34 \mathrm{~V}$ due to the absence of the oAP monomer, indicating that the redox peaks have been generated during the oxidation process of the oAP. To elucidate the effect of MWCNTs on the property of PoAP films, electrochemical performance of composite films was evaluated by carrying out cyclic voltammogram measurements in $0.5 \mathrm{M} \mathrm{NaClO}_{4} / \mathrm{HClO}_{4}$ (Figure $2 \mathrm{~b}$ ). The cyclic voltammograms of the MWCNTs/PoAP films show a higher background current in the potential sweep. The peak current of a MWCNTs/PoAP composite is stronger than that of pure a PoAP film. The voltammetric behavior of both films is similar and shows capacitive-like responses. A comparison between the voltammograms of MWCNTs/PoAP and pure PoAP electrodes demonstrates that the composite not only shows a higher background current in the potential sweep but also there exists faradic

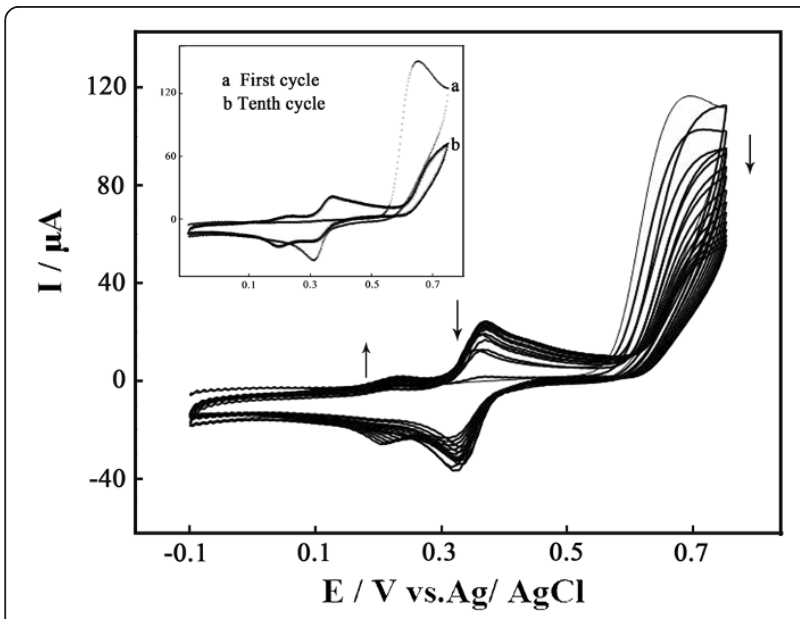

Figure 1 Cyclic voltammograms of oAP electropolymerization in $2 \mathrm{mM}$ oAP and $1 \%$ MWCNTs in $0.05 \mathrm{M} \mathrm{NaClO}{ }_{4} / \mathrm{HClO}_{4}$ with 0.01 M DBSA. Scan rate: $0.1 \mathrm{~V} \cdot \mathrm{s}^{-1}$. Inset: The first and tenth cycle of cyclic voltammograms of oAP electropolymerization.

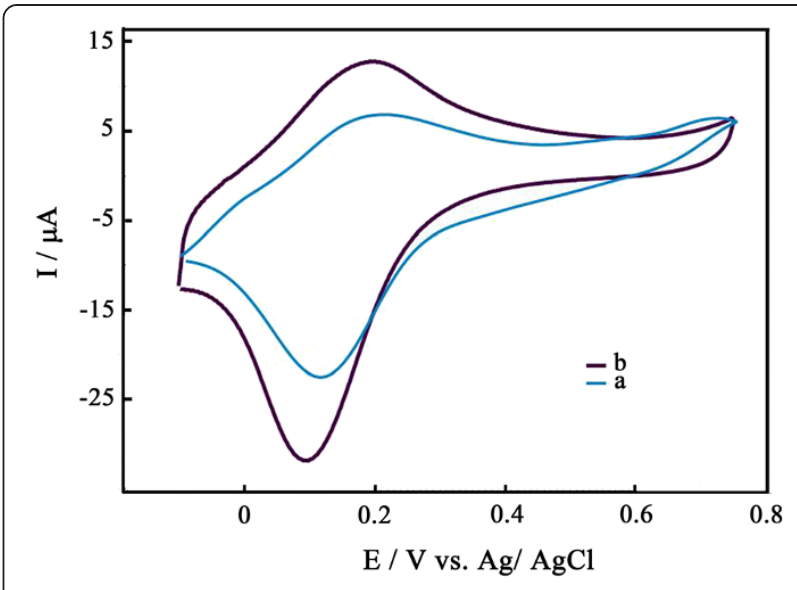

Figure 2 Cyclic voltammograms of (a) PoAP/PtE and (b) MWCNTs/PoAP/PtE obtained in $0.5 \mathrm{M} \mathrm{NaClO} / \mathrm{HClO}_{4}$ solution. Scan rate: $100 \mathrm{mV} \cdot \mathrm{s}^{-1}$

currents, which are due to the participation of the loaded MWCNTs. Owing to the higher currents in the cyclic voltammogram of the MWCNTs/PoAP, a larger capacitance for capacitors equipped with MWCNTs/PoAP is predictable and the MWCNTs/PoAP composite acts as a supercapacitor with a specific capacitance of $395 \mathrm{~F} \cdot \mathrm{g}^{-1}$. The specific capacitance was determined by the cyclic voltammetry technique. The specific capacitance $(S C)$ was calculated from the cyclic voltammogram, according to the following equation [20]:

$$
S C=\frac{2 i}{s m},
$$

where $i$ is the average cathodic current, $s$ denotes the applied potential sweep rate, and $m$ is the mass of each active material on the electrode.

Figure 3 shows the SEM micrographs of the pure PoAP and MWCNTs/PoAP composite. As it can be seen from Figure $3 \mathrm{a}$, PoAP/Pt has an incompact and rough structure. After the synthesis of MWCNTs/PoAP onto the surface of Pt (Figure 3b), a uniform and compact layer was obtained, indicating a strong interaction between the polymer and carbon nanotube [21]. It was clearly observed that the MWCNTs/PoAP composite (Figure $3 b$ ) has a larger surface area in comparison with PoAP/Pt films. The nanocomposite film formed a homogenous and three-dimensional film structure.

\section{Electrocatalytic oxidation of oxytetracycline}

The linear sweep voltammogram of different electrodes in a solution of oxytetracycline are shown in Figure 4. It can be seen that the oxytetracycline peak at the bare platinum electrode is relatively weak (Figure 4a) which is due to the slow electron transfer. Figure $4 \mathrm{~b}$ shows the oxidation of oxytetracycline on PoAP/Pt electrode. It is obvious that the presence of the PoAP film leads to the great reinforcement 

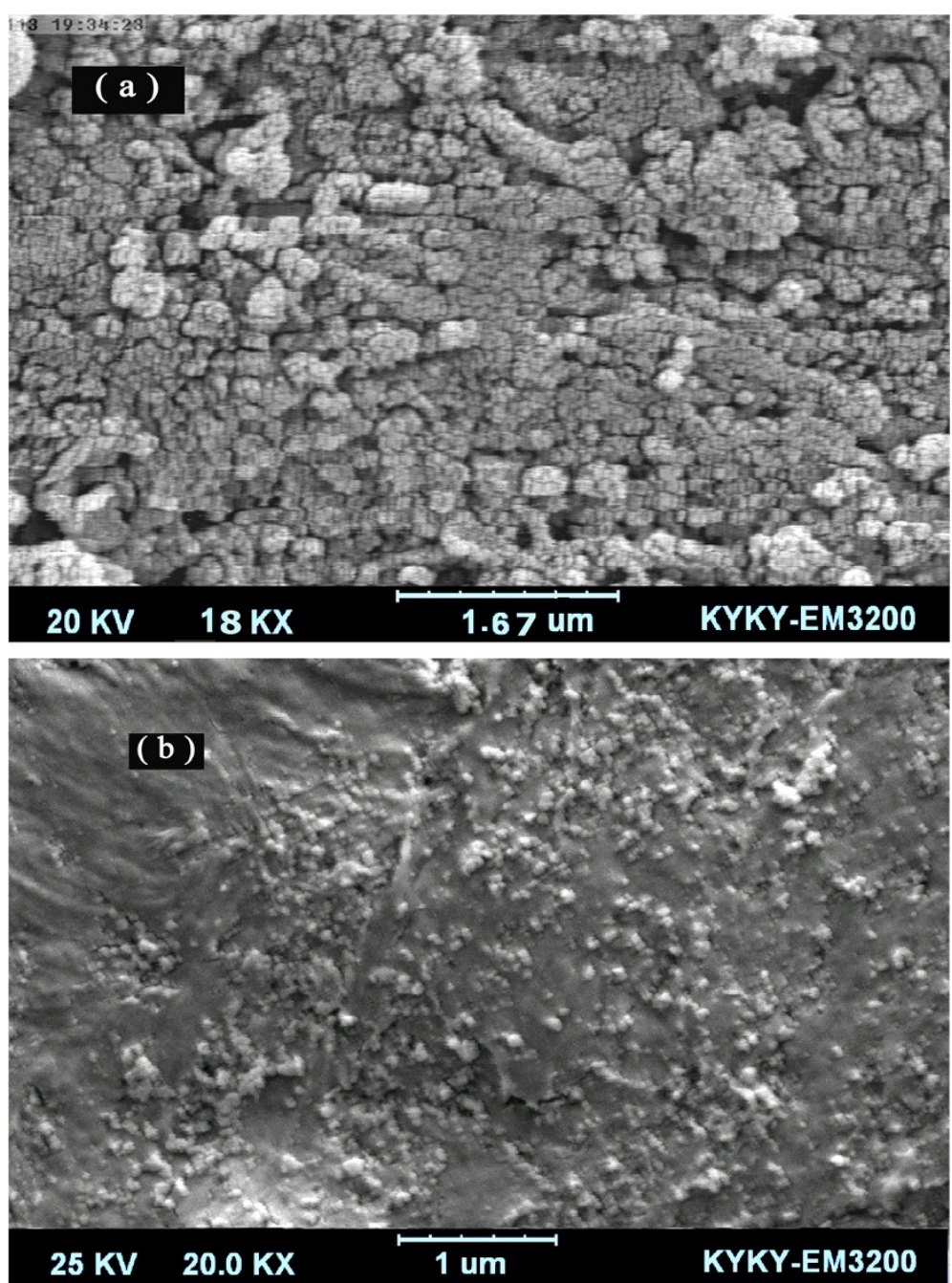

Figure 3 SEM micrographs of pure PoAP (a) and MWCNTs/PoAP nanocomposite (b).

of the anodic peak. The presence of MWCNTs on the bare electrode improves the electrocatalytic oxidation of oxytetracycline (Figure 4c). In comparison with other electrodes, MWCNTs/PoAP/Pt electrode exhibits better electrochemical response (Figure 4d). Therefore, the combination of PoAP and MWCNTs results in the increase of the oxytetracycline current response.

To some extent, the current of oxytetracycline is dependent on the thickness of MWCNTs/PoAP composite, which can easily be adjusted by controlling the number of potential cycles during the electropolymerization. The maximum anodic peak current of oxytetracycline is observed when 50 potential cycles were used for the fabrication of modified electrode (Figure 5) and then the electrochemical response decreased. So, the number of cycles was found to affect the sensitivity and linearity of the sensor. This indicates that increasing the MWCNTs/PoAP composite thickness after a specified extent leads to the reduced transfer

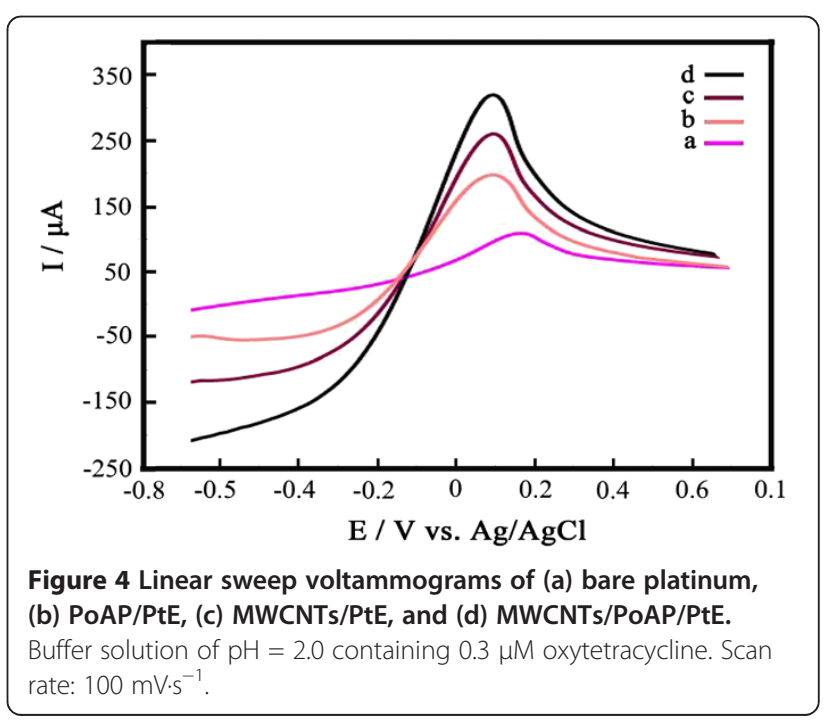




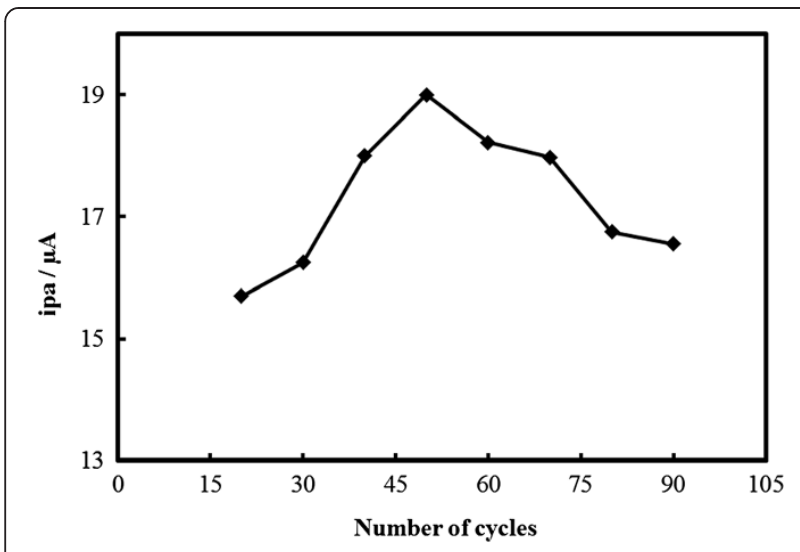

Figure 5 Dependence of anodic peak current $\left(i_{\mathrm{pa}}\right)$ of MWCNTs/ PoAP/PtE on number of potential cycles during electropolymerization of composite.

rate of electron in the film. Thus, 50 cycles of electropolymerization for fabrication the MWCNTs/PoAP composite was selected for this study and it exhibited favorable analytical performance.

For further investigation, the dependence of the anodic peak current $\left(i_{\mathrm{pa}}\right)$ of oxytetracycline with scan rate $(v)$ was also studied for MWCNTs/PoAP/PtE. As shown in Figure 6, the response of the MWCNTs/PoAP/PtE to oxytetracycline increases as the scan rate increases up to $70 \mathrm{mV} \cdot \mathrm{s}^{-1}$ and then decreases. The MWCNTs/ PoAP produced at a faster scan rate were found to form a loose film with a low recognition capacity. Therefore, the optimum scan rate has been found to be $70 \mathrm{mV} \cdot \mathrm{s}^{-1}$.

\section{Conclusion}

MWCNTs/PoAP composite were successfully fabricated through the electrochemical polymerization on the surface of platinum electrode. The SEM micrograph of the composite shows a porous morphology that wrapped on the surface of MWCNTs. The composite have good response to oxytetracycline at low potential. MWCNTs and the cross-linked structure of PoAP exhibit an electrocatalytic activity for the oxidation of oxytetracycline, which greatly facilitated the electron-exchange between oxytetracycline and the electrode and decreased the overpotentials for the oxidation of oxytetracycline. The sensor produced several advantages over the conventional, including low cost, sustainable electrocatalytic ability. It shows a distinguished stability. The sensor opens a new doorway for the application of MWCNTs/ PoAP as analytical devices. It might find application as an amperometric sensor with good stability and reproducibility for the determination of oxytetracycline which is reported in our other research.

\section{Methods}

All materials (ortho-aminophenol, dodecyl benzene sulfonic acid sodium, perchloric acid, sodium perchlorate, and oxytetracycline) were with analytical grade (Merck, Whitehouse Station, NJ, USA). Typical MWCNTs with outer diameter in the range of 5 to $20 \mathrm{~nm}$ and lengths up to several hundreds of nanometers were used. Solutions were prepared using doubly distilled water. Electrochemical studies were carried out in a onecompartment three-electrode cell powered by a Metrohm-Autolab potentiostat/galvanostat (model 12/ 30/302, Utrecht, The Netherlands). An Ag/AgCl electrode and a platinum foil with a large area were used as reference and auxiliary electrode, respectively. A MWCNTs/PoAP composite film was used as a working electrode. The morphology of the obtained films was characterized using a KYKY-EM3200 field emission

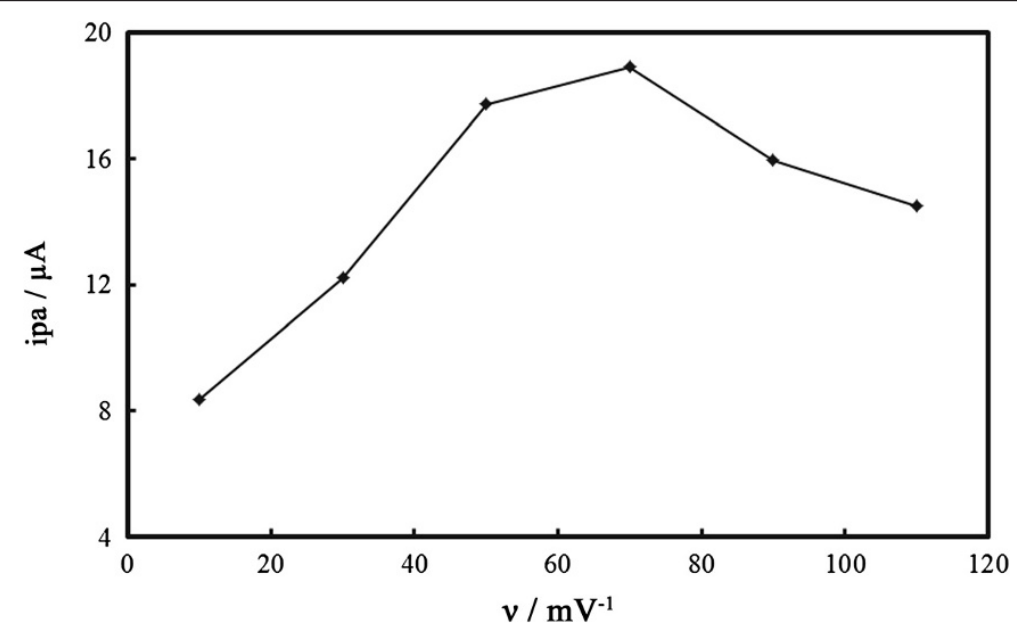

Figure 6 Effect of scan rate $(\mathbf{v})$ on anodic peak current $\left(\boldsymbol{i}_{\mathbf{p a}}\right)$ of MWCNTs/ PoAP/PtE. In a solution of pH $=2.0 \mathrm{containing} 0.3 \mu \mathrm{M}$ oxytetracycline. 
scanning electron microanalyzer (Beijing, China). The $\mathrm{pH}$ values of the solutions were determined using a $\mathrm{pH}$ meter (827, Metrohm, Riverview, FL, USA). Numerous amounts of purified MWCNTs were dispersed ultrasonically (Sigma-Aldrich-Bandelin Sonopuls ultrasonic homogenizer, Munich, Germany) into a dodecyl benzene sulfonic acid sodium solution. All the experiments were carried out at $22 \pm 1^{\circ} \mathrm{C}$.

\section{The preparation of the modified electrode (MWCNTs/ PoAP/ PtE)}

Bare platinum electrode was polished by $0.05 \mu \mathrm{m}$ alumina powder and then washed ultrasonically in water and ethanol, respectively. For the preparation of MWCNTs/polyortho-aminophenol (PoAP) composite film on platinum electrode, the aqueous micellar medium was prepared by dissolving $2.0 \mathrm{mM}$ oAP in an aqueous solution containing $0.2 \mathrm{mg} \mathrm{mL}^{-1}$ purified MWCNTs, $0.01 \mathrm{M} \mathrm{DBSA}$, and 0.05 M $\mathrm{NaClO}_{4} / \mathrm{HClO}_{4}$ ultrasonically for $10 \mathrm{~min}$. The MWCNTs/PoAP/PtE was formed by dipping PtE into the prepared mixture solution and then scanning in the potential range from -0.1 to $0.75 \mathrm{~V}$ at $0.1 \mathrm{~V} \cdot \mathrm{s}^{-1}$ for 50 cycles. The PoAP/PtE were prepared in the same way but without the addition of MWCNTs.

\section{Competing interests}

The authors declare that they have no competing interests.

\section{Authors' contributions}

NA carried out all of the experiments and analysis and drafted and rechecked the whole manuscript, and NBP rechecked the manuscript. Both authors read and approved the final manuscript.

\section{Acknowledgements}

The authors gratefully acknowledge the instrumental support from Payame Noor University.

Received: 6 August 2013 Accepted: 16 September 2013 Published: 01 Oct 2013

\section{References}

1. Firoz Babu, K, Subramanian, SP, Kulandainathan, M: Functionalisation of fabrics with conducting polymer for tuning capacitance and fabrication of supercapacitor. Carbohydr. Polym. 94, 487-495 (2013)

2. Hangarter, C, Chartuprayoon, N, Hernández, S, Choa, Y, Myung, N: Hybridized conducting polymer chemiresistive nano-sensors. Nano. Today. 8, 39-55 (2013)

3. Gaihre, B, Ashraf, S, Spinks, G, Innis, P, Wallace, G: Comparative displacement study of bilayer actuators comprising of conducting polymers, fabricated from polypyrrole, poly(3,4-ethylenedioxythiophene) or poly(3,4propylenedioxythiophene. Sens. Actuators. A: Physical. 193, 48-53 (2013)

4. Ehsani, A, Mahjani, MG, Jafarian, M, Naeemy, A: An electrochemical study of the synthesis and properties of multi-walled carbon nanotube/poly ortho aminophenol composites. Synth. Met. 161, 1760-1765 (2011)

5. Ayad, M, Abu El-Nasr, A: Anionic dye (acid green 25) adsorption from water by using polyaniline nanotubes salt/silica composite. J. Nanostr. Chem. (JNSC) 3(1), 3 (2012)

6. Shokrieh, MM, Saeedi, A, Chitsazzadeh, M: Mechanical properties of multiwalled carbon nanotube/ polyester nanocomposites. J. Nanostru. Chem. (JNSC) 3, 20 (2013)

7. Ojani, R, Raoof, J-B, Fathi, S: Poly (o-aminophenol) film prepared in the presence of sodium dodecyl sulfate: application for nickel ion dispersion and the electrocatalytic oxidation of methanol and ethylene glycol. Electrochim. Acta. 54, 2190-2196 (2009)
8. Hu, D, Peng, C, Chen, GZ: Electrodeposition of nonconducting polymers: roles of carbon nanotubes in the process and products. ACS. Nano. 4, 4274-4282 (2010)

9. Kan, K, Zhou, H, Li, C, Zhu, A, Xing, Z, Zhao, Z: Imprinted electrochemical sensor for dopamine recognition and determination based on a carbon nanotube/polypyrrole film. Electrochim. Acta. 63, 69-75 (2012)

10. Lota, G, Fic, K, Frackowiak, E: Carbon nanotubes and their composites in electrochemical applications. Energ. Environ. Sci. 4, 1592-1605 (2011)

11. Taleshi, F, Hosseini, AA: Synthesis of uniform MgO/CNT nanorods by precipitation method. J. Nanostruc. Chem. (JNSC) 3, 4 (2012)

12. Hesabi, M, Hesabi, M: The interaction between carbon nanotube and skin anti-cancer drugs: a DFT and NBO approach. J. Nanostr. Chem. (JNSC) 3(1), 22 (2013)

13. Farghali, AA, Bahgat, M, Elrouby WM, A, Khedr, MH: Decoration of multiwalled carbon nanotubes (MWCNTs) with different ferrite nanoparticles and its use as an adsorbent. J. Nanostru. Chem. (JNSC) 3, 50 (2013)

14. $\mathrm{Bi}, \mathrm{YH}$, Huang, ZL, Zhao, YD: The interface behavior and biocatalytic activity of superoxide dismutase at carbon nanotube. Biosens. Bioelectro. 21, 1350-1354 (2006)

15. Zhou, H, Cheng, X, Rao, L, Duan, Y: Poly (3, 4-ethylenedioxythiophene )/multiwall carbon nanotube composite coatings for improving the stability of microelectrodes in neural prostheses applications. Acta. Biomater. 9, 6439-6449 (2013)

16. Branzoi, F, Branzoi, V, Musina, A: Coatings based on conducting polymers and functionalized carbon nanotubes obtained by electropolymerization. Prog. Org. Coat. 76, 632-638 (2013)

17. Chopra, I, Roberts, M: Tetracycline antibiotics: mode of action, applications, molecular biology, and epidemiology of bacterial resistance. Microbiol. Mol. Biol. R. 65, 232-260 (2001)

18. $\mathrm{Mu}, \mathrm{S}$ : Electrochemical copolymerization of aniline and o-aminophenol. Synth. Met. 143, 259 (2004)

19. Zhang, L, Lian, J: Electrochemical synthesis of copolymer of aniline and oaminophenol and its use to the electrocatalytic oxidation of ascorbic acid. J. Anal. Chem. 611, 51-59 (2007)

20. Reddy, ALM, Ramaprabhu, S: Nanocrystalline metal oxides dispersed multiwalled carbon nanotubes as supercapacitor electrodes. J. Phys. Chem. C. 111, 7727-7773 (2007)

21. Gajendran, P, Saraswathi, R: Enhanced electrochemical growth and redox characteristics of poly(o-phenylenediamine) on a carbon nanotube modified glassy carbon electrode and its application in the electrocatalytic reduction of oxygen. J. Phys. Chem. C. 111, 11320-11328 (2011)

\subsection{6/2193-8865-3-76}

Cite this article as: Ajami and Bahrami Panah: One-step electrosynthesis of multi-walled carbon nanotube/poly-ortho-aminophenol composite film and investigation of its electrocatalytic properties. Journal Of Nanostructure in Chemistry 2013, 3:76

\section{Submit your manuscript to a SpringerOpen ${ }^{\mathcal{D}}$ journal and benefit from:}

- Convenient online submission

Rigorous peer review

- Immediate publication on acceptance

- Open access: articles freely available online

- High visibility within the field

- Retaining the copyright to your article

Submit your next manuscript at springeropen.com 